\title{
4MORE Uplink System Level Performance
}

\author{
M. Shateri ${ }^{\dagger}$, V. Monteiro ${ }^{\dagger \dagger}$, J. Rodriguez ${ }^{\dagger \dagger}$, A. Gameiro ${ }^{\dagger \dagger}$, R. Legouable ${ }^{\dagger \dagger}$, N. Ibrahim ${ }^{\dagger \dagger \dagger}$ \\ Centre for Communication Systems Research, Guildford, GU2 7XH, United Kingdom ${ }^{\dagger}$ \\ Instituto de Telecomunicaçoes, 3810-193 Aveiro, Portugal. Email jonathan@av.it.pt ${ }^{\dagger \dagger}$ \\ RD Rennes, 4 Rue du Clos Courtel BP 91226, 35512 Cesson Sevigne, France ${ }^{\dagger \dagger}$ \\ FT R\&D ,38/40 Rue du General Leclerc, 92794 Issy Les Moulineaux, France ${ }^{\dagger \dagger \dagger \dagger}$
}

\begin{abstract}
There is already significant research targeting B3G systems. The vision for this new generation includes provision of a broadband component for which various implementation technologies are being considered, one of them being MC-CDMA. The 4MORE project focuses at the system concept on techniques believed to be crucial to reach the broadband capabilities envisioned, namely Multiple Transmit Multiple Receive (MTMR) antennas and cross-layer design (L1 and L2). Specifically, the joint application of dynamic resource allocation with double Alamouti encoding and cross-layer signalling are considered to ensure seamless IP packet transport with broadband capabilities. The simulation results have shown that the 4MORE system can match the anticipated throughputs required for the cellular component of B3G systems.
\end{abstract}

Index Terms- 4MORE, MC-CDMA, MIMO, Dynamic Resource Allocation, Alamouti.

\section{INTRODUCTION}

$\mathrm{T}^{\mathrm{s}}$ he European vision for B3G systems incorporates a fully IP-based integrated architecture offering any service, at any time[1]. In order to accommodate future services requiring high capacity, a broadband component is envisioned with a maximum information bit rate of more than 2-20Mbps in a vehicular environment and possibly 50$100 \mathrm{Mbps}$ in indoor to pedestrian environments, using a 50 $100 \mathrm{MHz}$ bandwidth. One of the most promising technologies for this broadband component is Multi-Carrier Code Division Multiple Access (MC-CDMA), which allows combining the merits of OFDM (Orthogonal Frequency Division Multiplexing) with spread-spectrum techniques. In Japan [2] there has been in recent years a very strong emphasis on this technology including the realization of some field trials. At the European level and within the IST (Information Society Technologies) Program, the MATRICE [3] project has undertaken research in order to define and validate access and transmission concepts based on MC-CDMA technology to address the broadband component of future cellular systems.

In the 4MORE project [4], the intention was to complement the MATRICE and advance one step towards implementation though the design of a System on Chip (SoC) for a $4 \mathrm{G}$ terminal employing multiple antennas and based on MC-CDMA techniques. 4MORE objectives are to research, develop, integrate, and validate a cost effective, low power SoC solution for multi-antenna MC-CDMA mobile terminals based on the joint optimization of L1 (Layer 1) and L2 (Layer 2) functions. In this paper, we aim to address the system level aspect of the 4MORE air- interface in an attempt to quantify the broadband capabilities of the system thanks to the MIMO (Multiple Input Multiple Output) signal processing techniques introduced at L1. Furthermore, in order to utilise efficiently the 4MORE L1 spectral efficiency offered, we propose an uplink Dynamic Resource Allocation (DRA) scheme that ensures the seamless transport of variable size IP packets over the airinterface. The DRA constitutes link adaptation, H-ARQ (Hybrid- Automatic Repeat reQuest) type II and scheduling based on maxC/I (maximum Carrier-to-Interference ratio). The combination of these radio resource management entities ensures that variable size IP packets are mapped to a pre-defined set of radio block sizes, and are transported over the air-interface according to the H-ARQ protocol timing sequence. Furthermore, using cross-layer design techniques, we can ensure that each user is scheduled in a manner so as to ensure the highest average over-the-air (OTA) cell throughput.

In this paper, we aim to address the uplink system level performance for the 4MORE broadband air-interface based on the joint application of DRA with the latest technology in MIMO signal processing techniques at L1 using cross-layer signalling. This paper adopts the following structure: section II describes the 4MORE physical layer transmission chain; section III details the link level interface approach required to model the packet transmission quality at the system level; whilst the proposed uplink dynamic resource allocation scheme is given by section IV; simulation results are depicted in section $\mathrm{V}$; and finally the conclusion in section VI.

\section{PHYSICAL LAYER PLATFORM}

\section{A. Physical layer description}

Fig. 1 shows the synoptic view of the V0 (version 0) UL (uplink) 4MORE simulation chain that were used for generating the LUTs (Look-up-tables) for evaluating packet error at the system level. The transmitted bit vector is initially block encoded with the duobinary turbo code (DTC) channel coding with coding rates equal to $1 / 2,2 / 3$ or $3 / 4$; then the bits are interleaved and symbol mapping is performed following QPSK, 16QAM or 64QAM modulation.

The MC-CDMA technique allows several users to transmit simultaneously in the same bandwidth by using orthogonal Walsh-Hadamard spreading codes that lead to reduced Multiple Access Interference (MAI) in UL communication. The "Precoding" block is a Fast Hadamard Transform and simulates the SS-MC-MA (Spread Spectrum-Multi-CarrierMultiple Access) technique. Following the precoding stage, the symbols are subjected to Alamouti space time block 
coding [5], and OFDM modulated onto each transmit antenna. The MIMO channel is modelled according to the 3GPP/3GPP2 standard [6] and adapted for the 4MORE project at $5 \mathrm{GHz}$ in the $50 \mathrm{MHz}$ bandwidth. To achieve this, we have used an adaptation of the BRAN E channel model at $60 \mathrm{~km} / \mathrm{h}$ velocity.

After passing through the channel, at the reception, the OFDM demodulation is carried out by a simple and unique Fast Fourier Transform (FFT) applied to the sum of the $N u$ different users' signals. The received signal is STBC (Space Time Block Codes) MIMO decoded that consist in Alamouti's combining and equalization by using the frequency channel estimates. The resulting signal is despread, de-mapped, de-interleaved and decoded. For the decoding process the symbols at the input of the decoding process are weighted by the reliabilities of the propagation channel corresponding to the Log-Likelihood Ratios (LLR) of the channel. At the end of the simulation chain, we computed the PER (Packet Error Rate) of the transmission by comparing the input bit stream with the decoded bit stream. If, after the channel decoding at least one error occurs within the packet, then it is viewed as an erroneous packet.
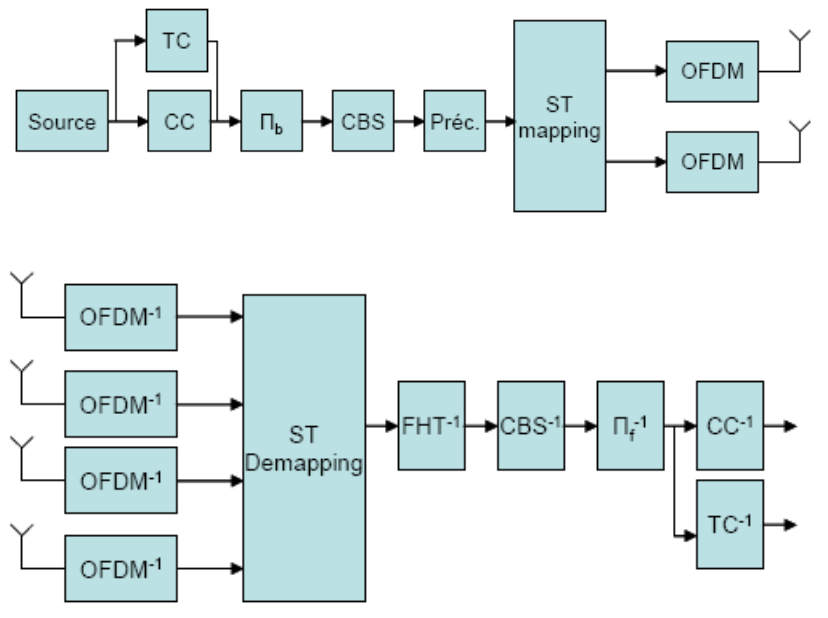

Fig. 1 UL 2x4 MIMO MC-CDMA 4MORE synoptic $\left(\mathrm{T}_{\mathrm{x}}\right.$ and $\mathrm{R}_{\mathrm{x}}$.)

\section{B. Frame structure}

The main goal of the proposed set of system parameters is to provide flexibility to the frame structure to allow either a $2 \times 2$ or a $2 \times 4\left(T_{x} \times R_{x}\right)$ system. The chosen frame represented by Fig. 2 is a $10 \mathrm{~ms}$ frame constituting 15 slots of $0.667 \mathrm{~ms}$, as defined for UMTS; although the MAC layer transmission time interval (TTI) is set to $2 \mathrm{~ms}$ duration. Each slot is dedicated either to uplink or downlink communications. The number of DL slots can be different from the number of UL slots allowing the operator to deal with the asymmetrical traffic requirements of the users. The allocation of UL and DL slots in the frame is determined by the network and can change at each new frame depending on traffic conditions. The base station (BS) regularly informs each MT (mobile terminal) in advance through a control channel about the allocation that has been chosen for the next frames.

Each UL and DL slot is composed of several symbols along the frequency and time dimensions of the OFDM frame. These symbols may be synchronisation or pilot symbols (denoted by $\mathrm{P}$ in Fig. 2) or data symbols (denoted by D in Fig. 2). The duration of each symbol includes the duration of the OFDM symbol itself i.e. $T_{F F T}$, plus the duration of the OFDM guard interval, $T_{\Delta}$, which may carry a cyclic prefix.

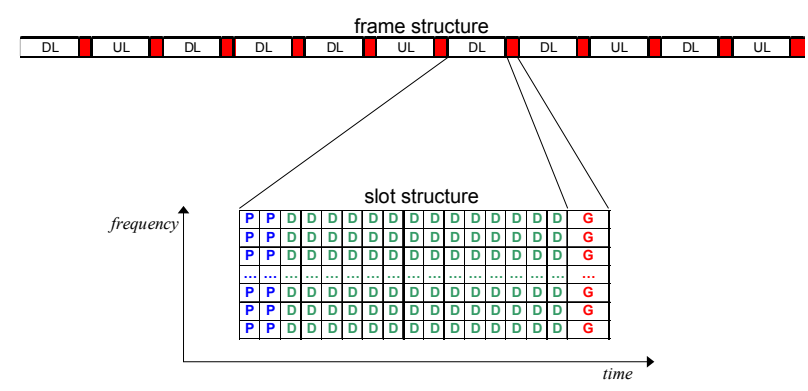

Fig. 2 Conventional 4MORE frame and slot structures.

The sampling frequency is denoted as $f_{S}$.

At the end of each slot, a TDD guard time is introduced to avoid interference between UL and DL communications. During this guard time, no information is transmitted thus introducing spectral efficiency losses; mainly when 2 consecutive slots are dedicated to the same transmission link. The duration of this TDD guard time $T_{G}$ is related to the cell range.

\section{1) Time frame structure}

For the uplink 2x4 MIMO MC-CDMA transmission mode:

- One OFDM symbol per slot is devoted to synchronisation $(\mathrm{S})$

- Full OFDM pilot symbols are inserted in the slot to regularly estimate the channel at the moving terminal $(\mathrm{P})$

The chosen time structure for the uplink is represented in Fig. 3.

\section{S8EPDDD DDDDDIDDDDPFDDDDIDDDDBDDDPG \\ Fig. 3 Timeslot structure for the uplink allowing $\mathrm{S}_{\mathrm{FT}}=4$ with 2 hopping areas.}

2) Frequency frame structure

From the number of pilot sub-carriers required for efficient common phase error (CPE) correction, the frequency structure of each OFDM data symbol depicted in Fig. 4 has been obtained. Among the 672 modulated subcarriers, each user transmit over a subchannel composed of 28 adjacent subcarriers. These 28 subcarriers are composed of $N_{D}=24$ subcarriers for useful data symbol transmission and $N_{P}=4$ pilots for $\mathrm{CPE}$ used for frequency offset compensation. Between each subchannel is inserted $N_{Z}=1$ null subcarrier for subchannel isolation to avoid intercarrier interference between users. So, the spectrum is subdivided into $N_{B}=24$ subchannels allowing up to 24 simultaneous users to transmit.

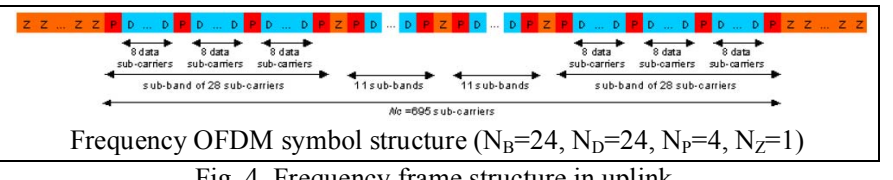

THE COMPLETE SET OF FRAME RELATED PARAMETERS ARE GIVEN BY Table I [7]. 
TABLE I

SYSTEM PARAMETERS

\begin{tabular}{lcc}
\hline \hline Quantity & Symbol & Value \\
\hline Channelisation bandwidth & $\mathrm{B}_{\mathrm{C}}$ & $50 \mathrm{MHz}$ \\
Max delay spread & $\Delta$ & $4 \mu \mathrm{s}$ \\
Cell radius & $\mathrm{R}$ & $<1 \mathrm{~km}$ \\
FFT size & $\mathrm{N}_{\mathrm{FFT}}$ & 1024 \\
Slot duration & $\mathrm{T}_{\text {slot }}$ & $0.667 \mathrm{~ms}$ \\
Physical Layer Frame duration & $\mathrm{T}_{\text {frame }}$ & $10 \mathrm{~ms}$ \\
Sampling frequency & $\mathrm{f}_{\mathrm{S}}=1 / \mathrm{T}_{\mathrm{S}}$ & $61.44 \mathrm{MHz}$ \\
Number of OFDM symbols per slot & $\mathrm{N}_{\mathrm{T}}$ & 32 \\
Guard interval in samples & $\mathrm{N}_{\Delta}$ & 256 \\
UL/DL guard time in samples & $\mathrm{N}_{\mathrm{G}}$ & 1280 \\
Number of UL modulated sub- & $\mathrm{N}_{\mathrm{C}}$ & 695 \\
carriers & $\mathrm{B}_{\mathrm{O}}$ & $41.7 \mathrm{MHz}$ \\
Occupied bandwidth & $\mathrm{N}_{\mathrm{D}, \mathrm{Symbol}}$ & 24 \\
Number of UL OFDM data symbols & $\mathrm{N}_{\mathrm{P}, \mathrm{Symbol}}$ & 4 \\
Number of UL OFDM pilot symbols & $\mathrm{N}_{\mathrm{D}}$ & 24 \\
Number of UL data sub-carriers per & & 4 \\
sub-band & $\mathrm{N}_{\mathrm{P}}$ & 8 \\
$\begin{array}{l}\text { Number of DL pilot sub-carriers per } \\
\text { sub-band }\end{array}$ & $\mathrm{S}_{\mathrm{F}}$ & \\
Max. Spreading factor in time (UL) & & \\
\hline \hline
\end{tabular}

\section{4MORE Data Rates}

Table II shows the available data rates on the 4MORE V0 UL. These are the MCS (Modulation Coding schemes)/transport formats that have been considered for LUT generation, and which are also used by the link adaptation unit at the MAC layer for resource allocation.

TABLE II

DATA RATES AND SPECTRAL EFFICIENCY FOR DIFFERENT MODULATION AND CODING RATES

\begin{tabular}{|c|c|c|c|}
\hline $\begin{array}{c}\text { DTC rate } \\
\text { and modulation }\end{array}$ & $\begin{array}{c}\text { Bit rate per user } \\
(\mathrm{Mbit} / \mathrm{s})\end{array}$ & $\begin{array}{c}\text { Total bit rate } \\
(\mathrm{Mbit} / \mathrm{s})\end{array}$ & $\begin{array}{c}\text { Spectral } \\
\text { efficiency }\end{array}$ \\
\hline \hline 1/2 QPSK & 0.86 & 20.7 & 0.5 \\
1/2 16QAM & 1.7 & 41.5 & 1 \\
1/2 64QAM & 2.6 & 62.2 & 1.5 \\
\hline 2/3 QPSK & 1.2 & 27.7 & 0.66 \\
2/3 16QAM & 2.3 & 55.3 & 1.33 \\
2/3 64QAM & 3.5 & 83.0 & 2 \\
\hline 3/4 QPSK & 1.3 & 31.1 & 0.75 \\
3/4 16QAM & 2.6 & 62.2 & 1.5 \\
3/4 64QAM & 3.9 & 93.3 & 2 \\
\hline
\end{tabular}

QPSK, 16QAM and 64QAM modulations associated with duobinary turbo code (DTC). Spectral efficiency and useful data rates attainable per user for each modulation/coding scheme.

\section{LINK LEVEL INTERFACE}

In $4 \mathrm{MORE}$, the resource allocation is very dependent on the channel evolution since the link adaptation and the packet scheduling uses channel state information to exploit the channel variability to improve packet transmission reliability. Thus, in order to be able to simulate accurately the effect of these functionalities, the Actual Value Interface (AVI) is considered. In contrast to WCDMA for which each coded symbol is spread over the whole bandwidth, in OFDM (and MC-CDMA system with reduced spreading factor) system, each symbol can be seen as mapped on a different sub-carrier or set of sub-carriers. Thus each codeword symbol experiences a different channel condition and therefore the instantaneous mean channel value can not be considered as a reliable quality metric; for this case another quality metric is considered: the Exponential
Effective SINR.

\section{A. EESM (Exponential Effective SINR Mapping) methodology}

The transmission of a coded block over different sets of sub-carriers results in a number of SINR measures that equals the number of sub-carriers sets, which can be quite high. Hence, data compression is mandatory. The coded symbol SINR can be given by:

$\operatorname{SINR}_{p}=G_{\text {actual }}\left(\sum_{m=0}^{L-1}\left|h_{m, p}\right|^{2}\right) / L$

where $G_{\text {actual }}$ is the actual geometry; $h_{m, P}$, is the complex amplitude of the $m^{\text {th }}$ sub-carrier in the $p^{\text {th }}$ subcarrier set; and $L$ is the spreading factor (MC-CDMA system)

The set of coded symbols SINRs are mapped to a single value named the Effective SINR value. This value can be used to match AWGN LUTs. The EESM [8] expression determines how the Effective SINR is obtained from the multiple SINR's on the different subcarriers:

$\operatorname{SINR}_{\text {eff }}=-\beta \ln \left(\frac{1}{P} \sum_{p=1}^{P} e^{-\frac{S I N R_{p}}{\beta}}\right)$

where $\beta$ is to be optimized for every link mode (MCS) based on link level simulation results.

\section{B. Interface Calibration}

The LUTs employed in conjunction with the studied interfaces are obtained from link-level simulations using a transmission over AWGN channels. Such LUT have to be generated for each employed MCS. A large number of BLER (Block Error Rate) simulation points have to be obtained with the frequency selective channel model to calibrate the interface for each MCS. For each of the $\mathrm{N}_{\text {sim }}$ simulated BLER points lying in the range of interest, a predicted BLER, denoted by $\operatorname{BLER}_{\text {pred,k }}(\beta)$ is obtained for each simulated point $k$. $\operatorname{BLER}_{\text {pred, } \mathrm{k}}(\beta)$ is then compared to the BLER $_{\mathrm{k}}$ obtained by simulation and $\beta$ can be optimised by attaining the least squares fit between the simulated and the predicted BLER points. The cost function to be minimized is:

$f_{\beta}=\sum_{k=1}^{N_{\text {sim }}}\left|\mathrm{BLER}_{\text {red } k}(\beta)-\mathrm{BLER}_{k}\right|^{2}=\left.\sum_{k=1}^{N_{\text {sim }}} \Delta \mathrm{BLER}_{\kappa}\right|^{2}$

The remaining $\triangle \mathrm{BLER}$ for the optimised value of $\beta$ can be used as an indicator of the quality of the calibrated Link to System (L2S) interface.

\section{EESM with MIMO}

The EESM approach can be directly extended to orthogonal MIMO schemes, e.g. $2 \times 2$ and $4 \times 2$ Alamouti Receiver Diversity, used in 4MORE. These techniques are known to enhance the channel quality link but keep the throughput unchanged in order to preserve orthogonality. As an example, after despreading, the SINR at the output of the $2 \times 2$ Alamouti scheme in Fig. 5 can be written as:

$\operatorname{SINR}_{p}=G_{\text {actual }}\left(\sum_{m=0}^{L-1}\left|h_{1, m, p}\right|^{2}+\left|h_{2, m, p}\right|^{2}+\left|h_{3, m, p}\right|^{2}+\left|h_{4, m, p}\right|^{2}\right) / L$ 


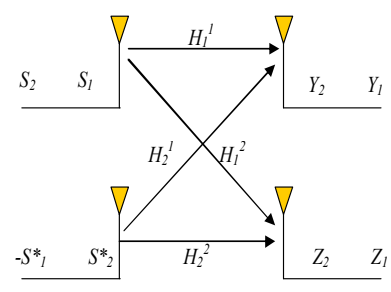

Fig. 5 2x2 Alamouti MIMO scheme

And thus the methodology is kept the same. Moreover, no need for further calibration in case of orthogonal MIMO scheme since it considered only an enhancement of the channel link quality. Since the EESM objective is to match all kind of channel variations with the AWGN behaviour through the $\beta$ variable and (2), the new MIMO channel quantity resulting from the Alamouti combination can be seen as an instance of the possible channel realizations.

\section{UPLINK DYNAMIC RESOURCE ALLOCATION}

The base station allocates radio resources dynamically according to a four frames cycle mechanism:

- Phase A1: The BS computes the Resource Allocation map to be used for data transmission during the phase C1.

- Phase B1: The BS broadcasts the Resource Allocation map (except the power information) determined during the phase A1, so that all users in the cell have the information required to modulate the packets they will transmit during the Phase $\mathrm{C} 1$.

- Phase C1: The MS sends data according to the Resource Allocation map determined during phase A1.

- Phase D1: The MS receives ACK (Acknowledgement) messages from users having received their packets successfully during phase $\mathrm{C} 1$, and receives NACK (Negative Acknowledgement) from BSs having received their packets un-successfully during phase $\mathrm{C} 1$. These received messages will be used in the next phase A1 to select users to be scheduled in the following phase $\mathrm{C} 1$.

We define four different Hybrid ARQ (HARQ) channels numbered from 0 to 3 . Each HARQ channel corresponds to one TDMA channel within a four frame cycle. Equally, we define four HARQ processes per MS, numbered from 0 to 3 . The HARQ Process number $n$ of mobile $k$ is synchronized with the HARQ channel $n$ of the base station. As shown by Fig. 6, the HARQ channels of the base station are time multiplexed, enabling the base station to achieve continuous transmission.

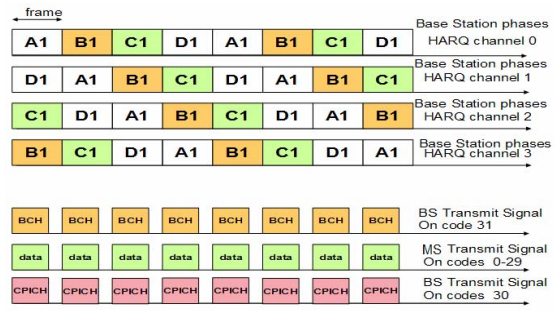

Fig. 6 HARQ data transmission cycle

The proposed DRA will always try to select the MS having the best propagation conditions so as to maximize cell throughput. Data blocks will be prioritized or discarded according to the time they have spent waiting in the queues.
DRA takes into account the following resource allocation constraints:

- Multiple users can transmit simultaneously over different sub-bands during one sub-frame, but each MS can have only one active HARQ process.

- Open loop power control performed for deciding on Pdata, allocated to data transmission

- Perfect control signalling is assumed in downlink and uplink.

The determination of the resource allocation by the DRA during the current sub-frame requires several steps:

\section{A. Updating Parameters DRA parameters}

1) Processing ARQ messages

Each MS identified by the MS ID, will update the DRA parameters according to the ARQ messages received during the previous sub-frame. Therefore, there are several cases to distinguish: If an MS ID has received an ACK for the active ARQ, then the ARQ process is de-activated; However, if an MS ID has received a NACK for the active ARQ process, then the priority timer is activated. Thus, if the MS ID has not been scheduled within the priority time duration then its priority is set to high. Furthermore, if the NACK corresponds to a first transmission Non-Acknowledgement then the packet discard timer is set. If the packet has not been scheduled within this time, the packet is discarded.

2) Updating priority levels

For each active ARQ process of each MS ID, if the data block of the ARQ process has been waiting for a retransmission for more than priority time, its priority must be set to high. The priority counter is also de-activated. For each un-active ARQ process of each MS ID, if the queue is not empty, then its priority must be set to low.

\section{3) Updating Priority List}

The two priority lists are updated. The ARQ processes that have a high level of priority are inserted into the high priority list. The ARQ process with a low level of priority and that are waiting for a packet to be transmitted are included in the low priority list.

\section{B. Packet Scheduling}

If the high priority list is not empty, then the ARQ process corresponding to the MS having the best SIR among the ARQ process of the list are selected. Otherwise, if the low priority list is not empty, the ARQ process corresponding to the MS having the best SIR among the ARQ process of the low priority list is selected. Otherwise, no ARQ process is scheduled. The scheduled MS ID is then allotted to the link adaptation unit that chooses MCS type according to the channel reliability and the available transmission formats available which are given by Table II. There are two cases to consider: if the selected MS ID and ARQ process requires a re-transmission, then the same MCS as for the first transmission is selected and the retransmission counter is incremented; if the selected MS ID and ARQ process requires a first transmission, then the number of bits that can be mapped onto one RU is deduced and the required number of bits from the user queue are withdrawn. Thus a subsequent packet concatenation/segmentation unit ensures that the variable size IP packets are encapsulated within the predefined radio block sizes; padding bits are used to ensure all radio blocks are filled. 


\section{Interaction with the physical layer.}

Finally the following inputs are sent to the physical layer for data transmission:

- Dynamic Resource Allocation table that provides the MS, and ARQ process scheduled for transmission on the current sub frame.

- HARQ table that gives the selected MCS, and the data block.

\section{Simulation Results}

The simulations were performed on a combined-dynamic snapshot simulator, where the mobiles are created at the beginning of each run, and remain active for the complete run duration. Furthermore, the pathloss and shadowing values remain constant within a run, whilst the fast fading is updated on each TTI (transmission time interval). Simulations were conducted in indoor environment where each run corresponds to 300 seconds of real time. The system level simulation parameters are given by Table III.

TABLE III

SYSTEM SIMULATION PARAMETERS

\begin{tabular}{|c|c|c|}
\hline Symbol & Quantity & Value \\
\hline$f_{c}$ & carrier frequency & $5 \mathrm{GHz}$ \\
\hline$P_{B S}$ & BS max. tx. Power & $17 \mathrm{~W}$ \\
\hline $\mathrm{P}_{\mathrm{MS}}$ & MS max. tx. Power & $0.5 \mathrm{~W}$ \\
\hline $\mathrm{N}_{\mathrm{o}}$ & Thermal Noise density & $-174 \mathrm{dBm} / \mathrm{Hz}$ \\
\hline $\mathrm{NF}_{\mathrm{m}}$ & MS Noise figure & $9 \mathrm{~dB}$ \\
\hline $\mathrm{CT}$ & Cell type & tri-sectored \\
\hline $\mathrm{d}_{\mathrm{is}}$ & Cell radius & $600 \mathrm{~m}$ \\
\hline $\mathrm{T}_{\mathrm{TI}}$ & TTI (Transmit Time Interval) & $2 \mathrm{~ms}$ \\
\hline $\mathrm{T}_{\mathrm{s}}$ & time slot duration & $0.667 \mathrm{~ms}$ \\
\hline $\mathrm{Ue}$ & no. of users per sector & 50 \\
\hline $\mathrm{CQI}_{\mathrm{d}}$ & $\begin{array}{l}\text { delay between CQI measurement and } \\
\text { use of CQI by the BS }\end{array}$ & $2 \mathrm{~F}_{\mathrm{d}}$ \\
\hline $\mathrm{CQI}_{\mathrm{m}}$ & time between two CQI measurements & $2 \mathrm{~F}_{\mathrm{d}}$ \\
\hline $\mathrm{Nb}_{\mathrm{tx}}$ & Max nb of re-transmissions & 3 \\
\hline S & user speed & $3 \mathrm{~km} / \mathrm{h}$ \\
\hline $\mathrm{G}_{\mathrm{ms}}$ & Mobile Antenna Gain & $0 \mathrm{dBi}$ \\
\hline $\mathrm{G}_{\mathrm{bs}}$ & BS Antenna Gain & $14 \mathrm{~dB}$ \\
\hline Ant $_{p}$ & tri-sectored antenna pattern & As in [9] \\
\hline $\mathrm{HP}_{\mathrm{t}}$ & high priority timer & $5 \mathrm{~F}_{\mathrm{d}}$ \\
\hline Dt & discard timer & $30 \mathrm{~F}_{\mathrm{d}}$ \\
\hline$\sigma_{\mathrm{s}}$ & shadowing standard deviation & $8 \mathrm{~dB}$ \\
\hline $\mathrm{ci}_{\mathrm{s}}$ & inter-site correlation & 0.5 \\
\hline $\mathrm{d}_{\mathrm{sh}}$ & shadowing de-correlation length & $20 \mathrm{~m}$ \\
\hline
\end{tabular}

The results obtained by simulations are based on the following assumptions:

- Scheduler operating in Max C/I and Round Robin modes

- No QoS (Quality of Service) guaranteed

In Fig. 7 we show the simulated average OTA and service sector throughput for different service types. Where the OTA is the total number of bits transmitted over the airinterface, and the service throughput is the number of successfully transmitted bits within the simulation time. It can be seen that the maximum possible theoretical OTA throughput per sector is $31.4 \mathrm{Mbps}$ if the link adaptation were to allocate the highest MCS consistently to all users which are scheduled. However, the maximum achieved OTA throughput per sector is about $30 \mathrm{Mbps}$. In practice even users close to the base station may experience a deep fade due to the statistical variation of the channel and thus forcing the maxC/I to schedule users which are closer to the cell edge with a lower order MCS. Since all users are served at least once by the BS, packets retransmissions are likely to occur by those users at the cell edge since there is inadequate signal quality to support even the lowest MCS option thus resulting in the discrepancy between service throughput and the OTA.

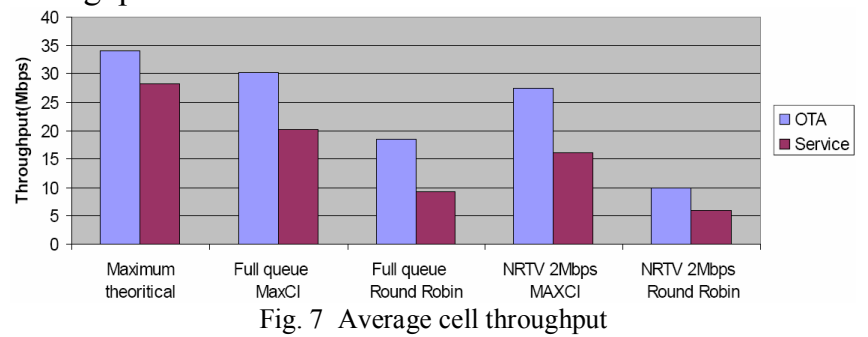

It was observed that $21 \mathrm{Mbps}$ service throughput can be achieved assuming a full queue traffic model with MaxC/I scheduling. The useful transmission bandwidth for the UL is $9.98 \mathrm{MHz}$ resulting in a spectral efficiency of $2.1 \mathrm{~b} / \mathrm{s} / \mathrm{Hz}$.

\section{CONCLUSION}

The 4MORE vision aims to provide candidate solution to the cellular component of $\mathrm{B} 3 \mathrm{G}$ systems by providing broadband services to the end-user in a seamless manner. 4MORE aims to provide $100 \mathrm{Mbps}$ throughput in the indoor environment using STBC in the form of Alamouti encoding with MIMO. In this paper we provide a system level analysis of the 4MORE transmission chain in order to evaluate whether we can effectively exploit the spectral efficiency offered by the physical layer, and taking into account the additional losses introduced by the MAC layer due to control signalling. Simulation results have shown that $2.1 \mathrm{~b} / \mathrm{s} / \mathrm{Hz}$ spectral efficiency can be achieved establishing 4MORE as a viable candidate air interface for beyond $3 \mathrm{G}$ cellular networks.

\section{ACKNOWLEDGMENTS}

The work carried out in the 4MORE project has been supported by the European Commission in the framework of FP6 with contract number IST-2002-507039, and the Treaty of Windsor Anglo-Portuguese Research Programme 0906II-AG.

\section{REFERENCES}

[1] E. Gustafsson, and A. Jonsson, "Always best connected," IEEE Wireless Communications, vol. 10, no. 1, pp. 49-55, Feb. 2003.

[2] H. Atarashi, N. Maeda, S. Abeta, M. Sawahashi, 'Broadband Wireless Access based on VSF-OFCDM and MC/DSCDMA,' Proc. of IEEE PIMRC 2002, vol. 3, pp. 992-997, Sept. 2002.

[3] The IST MATRICE project. Available: http://www.ist-matrice.org.

[4] The IST 4MORE project. Available: http://www.ist-4more.org.

[5] S. M. Alamouti. A simple transmit diversity technique for wireless communications. IEEE Journal on Selected Areas in Communications, 16(8):1451-1458, Oct. 1998.

[6] 3GPP2 TR 25.996, 2003-05, "Network spatial channel model for multiple input multiple output simulations (release 6)," 3GPP spatial channel AHG, sCM-134, Apr. 2003.

[7] J. Rodriguez et. al, "D2.1: Definition of framework for joint layer design and optimization", 4MORE WP2, Sept. 2004. http://www.ist4 more.org.

[8] Ericsson, "Effective SNR mapping for modelling frame error rates in multiple-state channels," 3GPP2-C30-20030429-010.

[9] France Telecom R\&D, "WCDMA-HSDPA system level simulator calibration", Montreal, Canada, Tech. Rep. 3GPP R1-04-0499, May 2004 